Epithelioid haemangioendothelioma (EHE) is a rare low-grade vascular neoplasm that is composed of mostly epithelioid cells. EHE may arise as a solitary tumour or in the form of multiple body lesions, and commonly occurs in soft tissues, liver, pleura, lung, peritoneum, lymph nodes, breast, and many other sites. EHE in the cranionasal region is extremely rare. There are very few reports of cases of skullbase EHE. We discuss an extremely rare presentation of an aggressive EHE that originated from the sellar region. Based on literature review, our patient is the first reported case of a giant solitary EHE with prepontine cistern invasion and abducens nerve encroachment mimicking a chondrosarcoma. We treated this rare tumour by near subtotal surgical excision with subsequent radiotherapy, considering that complete tumour resection with free margins in both cavernous sinus and clival region avoiding neural and vascular structure encroachment becomes technically difficult.

Key words: epithelioid haemangioendothelioma, vascular tumour, skull base, cranionasal.

Contemp Oncol (Pozn) 2018; 22 (2): 118-123 DOI: https://doi.org/10.5114/wo.2018.76235

\section{Giant cranionasal epithelioid haemangioendothelioma with invasive growth pattern mimicking a skull base chondrosarcoma}

\begin{abstract}
Kelvin Piña Batista ${ }^{1}$, Gonzalo Lepe Gómez², Eduardo Murias Quintana ${ }^{1}$, Aurora Astudillo ${ }^{1}$, Ivan Fernandez-Vega ${ }^{1}$, Belen Alvarez Fernandez ${ }^{1}$, Ana Cuellar-Martínez ${ }^{1}$, José Llorente-Pendás ${ }^{1}$, Veronica Rovira-Pereira ${ }^{1}$, Kenia Alvarez-Reyes ${ }^{3}$
\end{abstract}

${ }^{1}$ Hospital Universitario Central de Asturias, Spain ${ }^{2}$ Hospital Clínico Universitario de Valladolid, Spain ${ }^{3}$ Hospital Vital Álvarez Buylla, Spain

\section{Introduction}

Epithelioid haemangioendothelioma (EHE) is defined as a rare low-grade locally infiltrative vascular neoplasm with an epithelioid and histiocytoid aspect and metastatic spread potential. The term epithelioid haemangioendothelioma was first used by Weiss and Enzinger in 1982 in the context of soft tissue tumour [1-5]. The tumour displays transitional histological features between a well-differentiated haemangioma and angiosarcoma [2]. EHE can be present as either a solitary tumour or in the form of multiple body lesions, and commonly occurs in soft tissues, liver, pleura, lung, peritoneum, lymph nodes, breast, and many other sites. They are further subdivided into epithelioid, spindle cell, endovascular papillary, composite haemangioendothelioma, and Kaposiform [1].

EHE in the cranionasal region is extremely rare. Here, we describe and discuss a case of a 58-year-old female with a primary giant nasosinusal EHE invading through the orbit, the anterior skull base to the dura matter, and intradural extension into the prepontine cistern.

\section{Case report}

A 58-year-old female with a painless, progressive proptosis of her right eye that had developed four months earlier presented to the Emergency Department at the Universitario Central de Asturias Hospital. She also presented with a decreased uncorrected visual acuity, intermittent horizontal diplopia, and headaches. She denied any history of preceding trauma or ocular disease. There was no past medical, social, or family history of note. Physical examination revealed that the right pupil was dilated $(8 \mathrm{~mm})$ and had sluggish responses to direct and consensual pupillary light reflexes. Abducens palsy was noted on the right side. The remainder of the neurological examination was otherwise unremarkable.

The patient was referred to our Neurosurgical Department for further examinations. Initial head computed tomography (CT) demonstrated a heterogeneous mass centred in the right cavernous sinus and right sphenoid sinus that measured $6.5 \times 4.2 \times 3.7 \mathrm{~cm}$ with erosion of the optic canal, middle cranial fossa, clivus, and petro-occipital fissure and with protrusion to the sphenoid sinus and nasopharynx. Preoperative gradient-echo brain magnetic resonance imaging (MRI) scans revealed an expansile tumour with intracranial and extracranial components. Axial and sagittal T1-weighted imaging demonstrated a lobulated, hyperintense with heterogeneous isointensity to grey matter mass with its epicentre in the sphenoid sinus and right cavernous sinus. The tumour was 

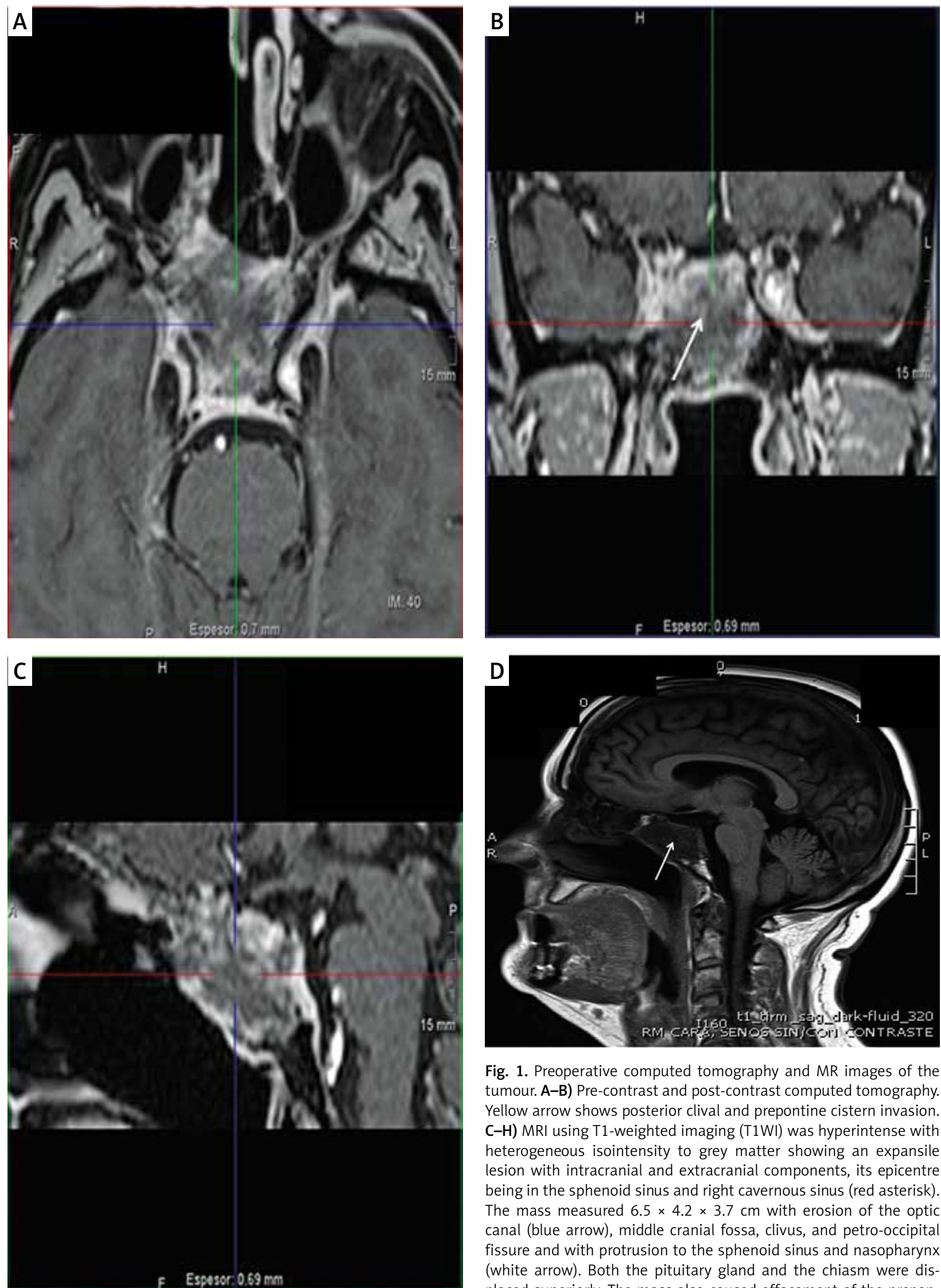

Fig. 1. Preoperative computed tomography and MR images of the tumour. A-B) Pre-contrast and post-contrast computed tomography. Yellow arrow shows posterior clival and prepontine cistern invasion. C-H) MRI using T1-weighted imaging (T1WI) was hyperintense with heterogeneous isointensity to grey matter showing an expansile lesion with intracranial and extracranial components, its epicentre being in the sphenoid sinus and right cavernous sinus (red asterisk). The mass measured $6.5 \times 4.2 \times 3.7 \mathrm{~cm}$ with erosion of the optic canal (blue arrow), middle cranial fossa, clivus, and petro-occipital fissure and with protrusion to the sphenoid sinus and nasopharynx (white arrow). Both the pituitary gland and the chiasm were displaced superiorly. The mass also caused effacement of the prepontine cistern with encroachment on the right internal carotid artery (ICA) and Dorello's canal, while T2WI showed a high hyperintensity along with partial isointensity to the grey matter, which suggested the presence of haemorrhage 

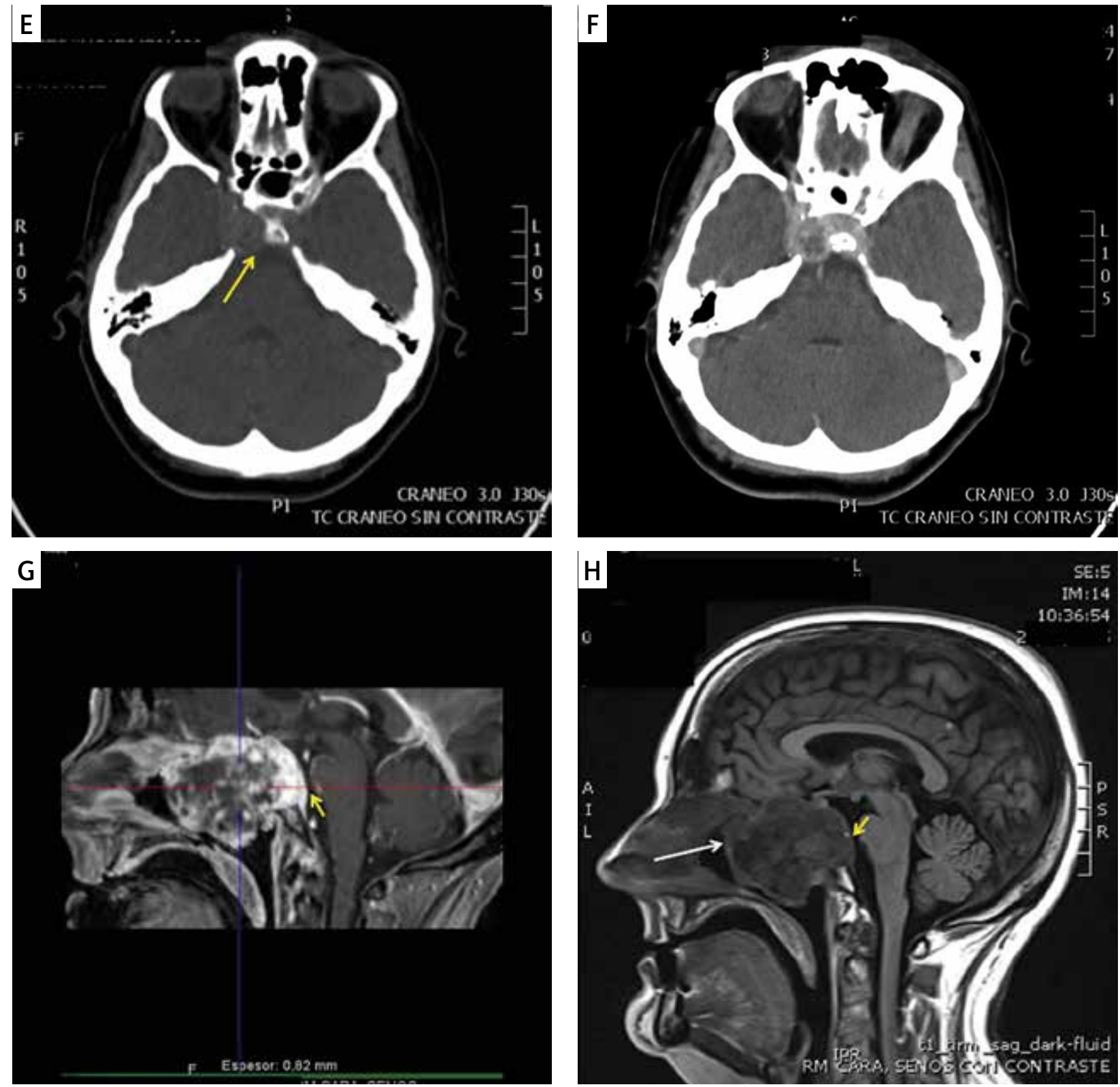

Fig. 1. Cont.

adjacent to the right temporal lobe (Figs. 1B, 1E, 1F, 1H). Both the pituitary gland and the chiasm were displaced superiorly (Figs. 1C, 1D). The mass also caused effacement of the prepontine cistern with encroachment on the right internal carotid artery (ICA) and Dorello's canal. T2-weighted images showed a high hyperintensity along with partial isointensity to the grey matter, which suggested the presence of haemorrhage (Fig. 1). Based on these radiological features of the lesion, a diagnosis of chondrosarcoma was determined.

The patient underwent a direct endoscopic endonasal transsphenoidal surgery with the aid of neuronavigation. Intraoperative Doppler ultrasound was used to identify the location of the right ICA. During the procedure, a haemorrhagic lesion was found to be attached to the sphenoid sinus walls. The tumour extended from nasopharynx towards the prepontine cistern, transgressing the dura. Tumour debulking was initiated centrally and progressed laterally. Due to the location of the lesion, it was determined

to be near subtotally resectable by the surgical team. There were no postoperative complications. Radiotherapy was administered to complement the surgical treatment.

Macroscopically, the tumour was solid and well demarcated, measuring $3 \times 4 \mathrm{~cm}$. On cut section the specimen was partially greyish.

Microscopically, haematoxylin and eosin-stained sections showed that neoplastic histoarchitecture was regular with growing and hyperplastic capillary channels. The neoplastic cytoplasm was scarce with nuclei having prominent and irregular nucleoli. Red cell inclusion appeared in the cytoplasm of neoplastic cells. The tumour cells were grouped in a fibromyxoid stroma. Immunostainings demonstrated expression of endothelium-related molecules as CD99, CD31, CD34, and anti-factor VIII, but negative for cytokeratin AE1/3. Ki-67 proliferation index was 10\% (Fig. 2). Finally, based on the above findings, the diagnosis was confirmed to be EHE. 

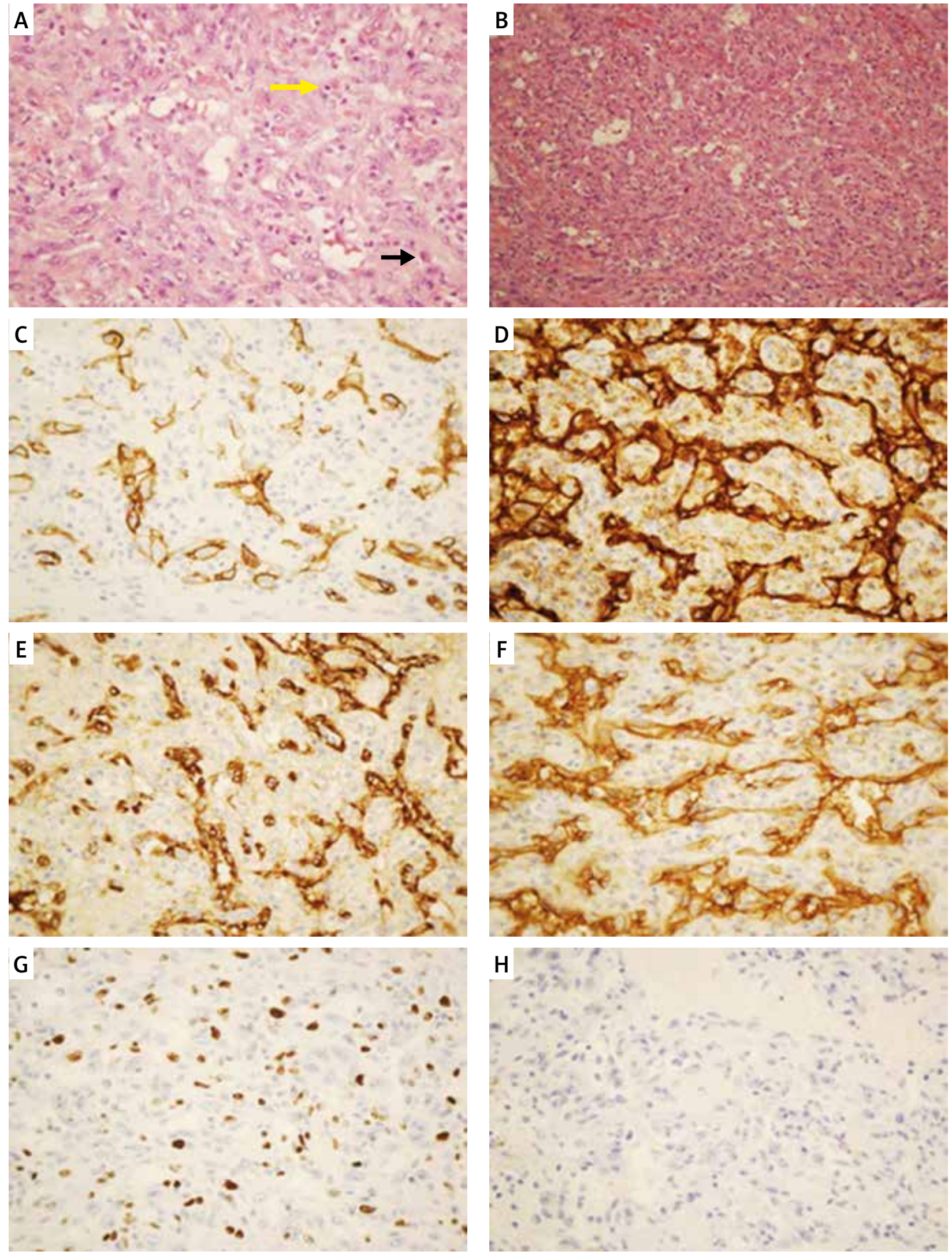

Fig. 2. Histopathological findings. A-B) Immunohistochemical staining showing positivity of the spindle to epithelioid tumour cells embedded in myxofibro-hyaline stroma (400x and 200x, respectively), demonstrating intracytoplasmic lumina (yellow arrow) with erythrocytes (black arrow). C, D, E, and F) Tumour cells were positive for CD34, CD31, Factor VIII, and CD99, respectively (400x). G) Ki67 proliferation index was 10\% (400x). H) The immunohistochemical stains revealed negativity for cytokeratin (400x) 

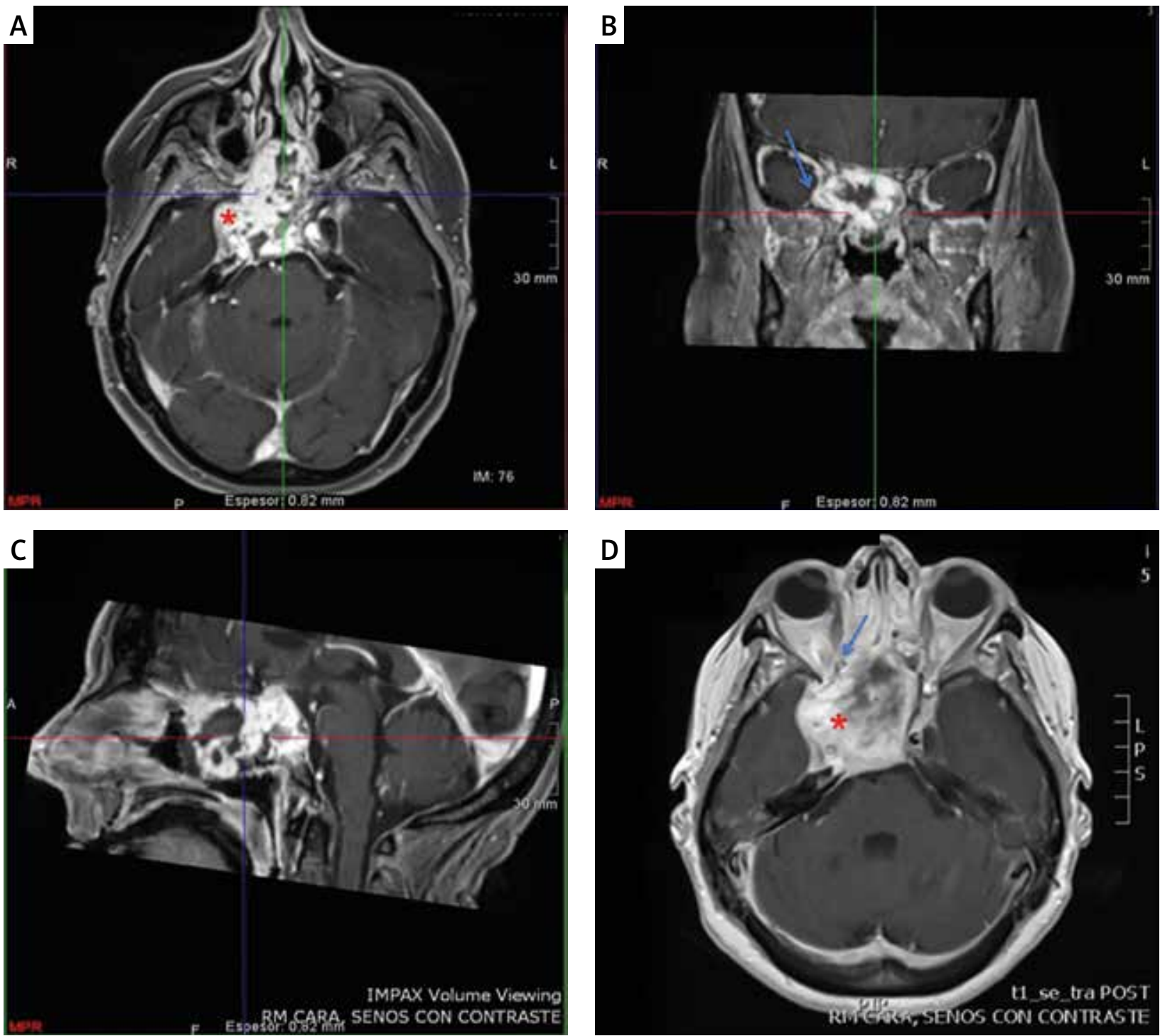

Fig. 3. Postoperative MRI showing minimal residual tumour (white arrow)

Recovery was uneventful, and the patient was discharged 14 days after surgery. Until now, the therapeutic approach has been satisfactory, and our patient is both symptom and recurrence free (Fig. 3) two years after resection of the tumour.

\section{Discussion}

Herein, we discuss an extremely rare presentation of an infiltrative EHE that originated from the sellar region. Based on literature review, our patient is the first reported case of a giant solitary EHE with prepontine cistern invasion, and abducens nerve encroachment. In the literature, five cases of EHE of the clival region have been described [6-10]. These very rare neoplasms account for only $1 \%$ of all vascular tumours. It most commonly originates from liver and lung, followed by the bone (about 14\%); however, skull base involvement is a particularly rare event. In addition, few cases of EHE are described in the nasal cavity in English literature [1, 2, 4, 11-13].

Microscopically, EHE cells grow forming intracellular luminal structures of various sizes, containing red blood cells inside $[3,4,8,12]$, which are also a characteristic feature of angiosarcomas [6, 14, 15]. However, angiosarcoma is unique with regard to its unusual pattern of irregular anastomosing vascular spaces lined by malignant endothelial cells. As shown in Figure 2, EHE is characterised by the presence of epithelioid cells with intracytoplasmic vacuoles [12], and atypical disposition in cords and nests [3] in a fibromyxoid stroma with very low metastatic potential. Histologically, definitive diagnosis of these tumours may be a challenge, and histological examination and immunohistochemical markers is needed to distinguish EHE from poorly differentiated carcinomas $[1,3,8,11]$. The differential diagnosis thus includes angiosarcoma, chordoid meningioma [8, 16], epithelioid haemangioma (EH), chordoma [17], and chondrosarcoma [18]. In this case, however, the results of immunohistochemical study excluded all of these tumours. Immunostaining for CD99, CD31, CD34, and factor VIII-associated antigen [1, 4, 15] is crucial. Because of vascular proliferation, differential diagnosis between angiosarcoma and EHE is required regularly. Angiosarcoma shows vascular differentiation with irregular channels, atypical mitotic, 
pleomorphic malignant cells, and necrosis. Approximately 30-50\% of epithelioid angiosarcomas show cytokeratin immunoexpression $[4,15,19]$. In contrast to the angiosarcomas, EHEs are a low-grade malignancy tumour with less metastatic potential than angiosarcomas.

Chordomas and chordoid meningiomas cells are supported by a chondroid stroma, and tumour cells demonstrate negative immunostaining for CD 31 and CD 34. On the other hand, chondrosarcomas must be taken into consideration. Chondrosarcomas represent the third most common malignant tumours of the bone and can be misdiagnosed as skull-base chordomas, being challenging lesions even for pathologists because of their strikingly similar morphology. Chondrosarcomas are composed of nested tumour cells with occasional vacuolisation embedded in a fibrous matrix $[8,17,20]$. Chordoma stains for EMA and cytokeratin. It has a much better prognosis than chondrosarcomas.

Epithelioid haemangioma $(E H)$ is a rare benign neoplasm of soft tissue with a good prognosis. Epithelioid haemangiomas are diagnosed mainly based on histopathological features of epithelioid endothelial cells containing a lot of cytoplasm with intracytoplasmic lumina, large nuclei, and prominent nucleoli $[1,2]$. EH is characterised by low mitotic index and absent nuclear atypia.

\section{Conclusions}

Primary EHE should be considered when there is a well-circumscribed capsule mass composed of epithelioid endothelial cells, arranged in short nests supported in a myxofibro-hyaline stroma, even though it is difficult to be diagnosed radiologically before surgery and it requires careful pathologic examination for definitive diagnosis. Furthermore, unfortunately EHE does not have distinctive radiologic characteristics.

According to the scarce literature published regarding EHE neoplasm in the skull base, the best therapeutic approach is complete surgical excision [4]. We treated this rare tumour by near subtotal surgical excision with subsequent radiotherapy, considering that complete tumour resection with free margins in both cavernous sinus and clival region avoiding neural and vascular structures encroachment becomes technically difficult. Furthermore, there are issues to be addressed regarding middle skullbase reconstruction $[4,7,11,21,22]$.

The authors declare no conflict of interest.

\section{References}

1. Fernandes AL, Ratilal B, Mafra M, Magalhaes C. Aggressive intracranial and extra-cranial epithelioid hemangioendothelioma: A case report and review of the literature. Neuropathology 2006; 26: 201-205.

2. Aniba K, Laghmari M, Lmejjati M, Ghannane H, Ait Benali S. A tragical paediatric case history of intraorbital and intracranial epithelioid hemangioendothelioma. Case Rep Neurol Med 2012; 396097.

3. Naqvi J, Ordonez NG, Luna MA, Williams MD, Weber RS, El-Naggar AK. Epithelioid Hemangioendothelioma of the Head and Neck: Role of podoplanin in the differential diagnosis. Head Neck Pathol 2007; 2: 25-30.
4. Sardaro A, Bardoscia L, Petruzzelli MF, Portaluri M. Epithelioid hemangioendothelioma: an overview and update on a rare vascular tumor. Oncol Rev 2014; 8: 1-10.

5. Weiss SW, Enzinger FM. Epithelioid hemangioendothelioma: a vascular tumor often mistaken for a carcinoma. Cancer 1982; 50: 970-981.

6. Adler B, Naheedy J, Yeager N, Nicol K, Klamar J. Multifocal epithelioid hemangioendothelioma in a 16-year-old boy. Pediatr Radiol 2005; 35: 1014-1018.

7. Medina M, Polo R, Vaca M, Alonso A, Cobeta I. Multifocal epithelioid hemangioendothelioma with massive lateral skull base involvement. Otol Neurotol 2015; 36: e67-69.

8. Ma SR, Li KC, Xu YQ, Wang YM, Ma WL, Li Q. Primary epithelioid hemangioendothelioma in the clival region: A case report and literature review. Neuropathology 2011; 31: 519-522.

9. Rushing EJ, White JA, D’Alise MD, Chason DP, White CL 3rd, Bigio EH. Primary epithelioid hemangioendothelioma. Clin Neuropathol 1998; 17: 110-114.

10. Watanabe T, Saito N, Shimaguchi H, Fujimaki H, Kamiya M, Nakazato Y, Sasaki T. Primary epithelioid hemangioendothelioma originating in the lower petroclival region: case report. Surg Neurol 2003; 59: 429-433; discussion 434.

11. Ogita S, Endo T, Nomura K, et al. Nasal cavity epithelioid hemangioendothelioma invading the anterior skull base. Surg Neurol Int 2016; 7: 53-57.

12. Patnayak R, Jena A, Reddy K, Chowhan A, Rao CM, Rukhamangadha N. Epithelioid hemangioendothelioma of nasal cavity. J Lab Physicians 2010; 2: 111-115.

13. Fletcher CDM, Bridge JA, Hogendoorn P, Mertens F (eds.). World Health Organization Classification of Tumours. Pathology and genetics of tumours of soft tissue and bone. IARC Press, Lyon 2013.

14. Miettinen M, Wang Z, Lasota J, Heery C, Schlom J, Palena C. Nuclear Brachyury Expression Is Consistent in Chordoma, Common in Germ Cell Tumors and Small Cell Carcinomas, and Rare in Other Carcinomas and Sarcomas. Am J Surg Pathol 2015; 39: 1305-1312.

15. Louis DN, Ohgaki H, Wiestler OD, Cavenee WK, Burger PC, Jouvet A, Scheithauer BW, Kleihues P. The 2007 WHO Classification of Tumours of the Central Nervous System. Acta Neuropathol 2007; 114: 97-109.

16. Wang L, Wu Z, Tian K, Li G, Zhang J. Clinical and Pathological Features of Intradural Retroclival Chordoma. World Neurosurg 2014; 82: 791-798.

17. Piña-Batista KM, Álvarez KY, Lopez FP, et al. Immunophenotypic features of dedifferentiated skull base chordoma: an insight into the intratumoral heterogeneity. Contemp Oncol 2017; 21: 267-273.

18. Sekhar LN, Pranatartiharan R, Chanda A, Wright DC. Chordomas and chondrosarcomas of the skull base: results and complications of surgical management. Neurosurg Focus 2001; 10: E2.

19. Hornick JL (Ed.). Practical Soft Tissue Pathology: A Diagnostic Approach. Elsevier/Saunders: Philadelphia 2013.

20. Barresi V, leni A, Branca G, Tuccari G. Brachyury: a diagnostic marker for the differential diagnosis of chordoma and hemangioblastoma versus neoplastic histological mimickers. Dis Markers 2014; 514753.

21. Zhu Y, Fan M, Pandey S, Liang W, Chang D. Multiple epithelioid hemangioedothelioma of the skull in a child. Medicine (Baltimore) 2016; 95: e4081-4083.

22. Pamir MN, Ozduman K. Analysis of radiological features relative to histopathology in 42 skull-base chordomas and chondrosarcomas. Eur J Radiol 2006; 58: 461-470.

\section{Address for correspondence}

\section{Kelvin Piña Batista}

Hospital Universitario Central de Asturias

Av. de Las Segadas 22, 4 a

33006 Oviedo, Spain

e-mail: pineappledr@gmail.com

Submitted: 20.04 .2018

Accepted: $\quad 21.04 .2018$ 